\title{
BERLIN VILLE VERTE ET DURABLE : UTOPIE OU RÉALITÉ ?
}

\author{
Boris Grésillon et Ares Kalandides
}

Au début de l'an 2020, le Sénat de Berlin (le gouvernement de la Ville-Land) s'est doté d'une "Charte de la ville verte de Berlin " (Senatsverwaltung für Umwelt, Verkehr und Klimaschutz, 2020).dans laquelle il formule les objectifs, les tâches et les mesures nécessaires pour protéger, renforcer et développer "la ville verte», même dans un Berlin en pleine croissance démographique : en dix ans, de 2009 à 2019, la population de la capitale allemande a crû de plus de 200.000 habitants, après deux décennies de quasi-stagnation - passant de 3440000 habitants en 2009 à 3670000 fin 2019.

Avec la Charte, un programme d'action pour la ville verte de Berlin 2030 a été adopté. Le programme d'action contient des projets, mesures et instruments spécifiques pour relever les défis formulés dans la Charte et atteindre les objectifs fixés. Ces derniers sont mis à jour, ajustés et évalués. La Charte s'inscrit dans une stratégie environnementale plus large, que Berlin poursuit depuis des décennies et qui comprend (entre autres) des programmes de transition énergétique et de protection du paysage urbain. Le Sénat de Berlin poursuit l'objectif à long terme de faire de Berlin une ville climatiquement neutre d'ici 2050. Avec cet objectif ambitieux de protection du climat, Berlin, comme de nombreuses autres métropoles internationales, répond aux dangers du changement climatique et apporte sa contribution à la réalisation des objectifs de I'Accord de Paris de 2015. Les objectifs de protection du climat de Berlin sont ancrés dans la «loi de Berlin sur la transition énergétique " de 2016 (Abgeordnetenhaus von Berlin, 2016). Cette loi attribue au secteur public un rôle moteur dans le domaine de la protection du climat. Cela se concrétise, entre autres, par des calendriers de rénovation des bâtiments publics et par un schéma directeur pour une administration neutre en CO2.

Le "programme de protection de l'énergie et du climat de Berlin 2030 " (Berliner Energie- und Klimaschutzprogramm, BEK 2030) contient les stratégies et mesures concrètes à mettre en œuvre sur la voie de la neutralité climatique. Il constitue ainsi le « calendrier » et l'instrument central de cette politique Berlin. Ces quelque 100 mesures représentent la diversité de la politique climatique à Berlin et abordent à la fois la protection du climat et l'adaptation aux conséquences inévitables du changement climatique (Senatsverwaltung für Umwelt, Verkehr und Klimaschutz, 2019).

Voilà pour ce qui est de l'arsenal législatif récent en matière écologique. Dans les faits, Berlin est façonné par les espaces verts comme peu d'autres métropoles en Europe. Sur une agglomération, il est vrai, immense $(881 \mathrm{~km} 2)$, les espaces ouverts et les espaces verts représentent près de $44 \%$ de la superficie de la Ville-Land. C'est une base importante pour l'attractivité de la capitale allemande et pour sa haute qualité de vie. 
La "stratégie du paysage urbain de Berlin » de 2011 a pour objectif de sécuriser cette " substance " dans une ville en pleine croissance et la développer dans la mesure du possible.

Si les politiques publiques berlinoises sont sensibles à l'environnement depuis plusieurs années, la force relative du parti écologiste Bündnis 90/ Die Grünen en est symptomatique, tant l'intensité que le sens de cette sensibilité ont évolué au fil des années. Dans cet article, nous utiliserons le terme de durabilité au sens de protection à long terme des ressources matérielles, économiques et sociales, dans les trois dimensions suivantes : environnement, société, économie. Nous suivons en cela l'utilisation du terme dans la stratégie de développement officielle de Berlin. (Senatsverwaltung für Stadtentwicklung und Umwelt, 2016)

Nous nous proposons de suivre cette évolution au cours des trois dernières décennies et de montrer les particularités de chaque phase, en insistant davantage sur la dernière. Nous suivrons largement les périodes électorales, car elles marquent des changements dans l'élaboration des politiques et des instruments de planification. En gros, ces phases vont de 1990 à 2001 (période où le parti chrétien-démocrate (CDU) gouverne avec son allié du SPD) ; de 2001 à 2011 (deux périodes de coalition dite rouge-rouge SPD-PDS), de 2011 à 2016 (coalition de centre-droit SPD / CDU) et de 2016 jusqu'à aujourd'hui, avec la coalition « rouge-rouge-verte » (SPD, Die Linke et Bündnis 90/ Die Grünen).

\section{0 - 2001 Les grands chantiers}

Les années 1990 sont marquées par les conséquences de la chute du Mur de Berlin et par le processus d'intégration du territoire de l'ex-RDA dans la République fédérale. Une restructuration de tout le pays a lieu. Berlin, en tant que nouvelle capitale, constitue symboliquement et matériellement l'épicentre de ces changements. En effet, c'est la décision de déplacer la capitale allemande de Bonn à Berlin en 1991, un processus qui a occupé la majeure partie des années 1990, qui marque cette décennie : le Bundestag (Parlement national) déménage en 1999 dans le bâtiment du Reichstag rénové par l'architecte Norman Foster ; il est flanqué d'un long bâtiment - le "Band des Bundes" ("la bande de la fédération") - abritant les bureaux et salles de réunion des députés (architectes : Axel Schultes et Charlotte Frank); en face de lui s'élève la nouvelle Chancellerie conçue par les mêmes architectes; et à proximité, disséminés dans le quartier central de Mitte, les principaux ministères, les grandes centrales politiques ainsi que les ambassades. La nouvelle géographie du pouvoir est en place.

Dans le même temps, la ville est devenue l'espace privilégié de larges débats sur la planification, avec des discussions à grande échelle sur le sens de l'architecture, la reconfiguration de l'espace urbain et des échanges tendus sur les priorités de la politique locale. Il existe en gros trois principaux domaines d'activité à l'époque : (1) Les activités de construction des nouvelles fonctions capitales, Parlement, chancellerie, 
ministères, etc. ; (2) Les investissements privés à grande échelle dans la construction de grandes parcelles vides, principalement mais pas exclusivement là où se trouvait le Mur de Berlin ; et (3) la rénovation de quartiers entiers, à la fois de la période de fondation dite Gründerzeit (fin XIXème et début XXème siècles) et des complexes d'habitation de masse préfabriqués industriellement construits en périphérie de Berlin-Est. Les grands projets de construction ont tous été soutenus par une refonte massive de l'infrastructure technique de la ville : nouvelles lignes de métro et de train, modernisation du chauffage urbain et de l'approvisionnement en énergie, nouvelles routes et autoroutes.

Le nouveau plan d'aménagement du territoire (Flächennutzungsplan ou FNP), qui a été élaboré en très peu de temps pour refléter les changements attendus de la ville en matière de développement, a été adopté par le parlement du Land en 1994. Ses principales dispositions étaient la restriction du développement ultérieur à l'intérieur de la ville afin d'éviter l'étalement urbain, le renforcement des logements en centre-ville, la protection des espaces verts (parcs, jardins et bois), la consolidation de la structure polycentrique de la ville au-delà des deux principaux centres de Berlin-Est et BerlinOuest, l'établissement de zones réservées à une éventuelle utilisation industrielle future (sachant que la ville a perdu 80\% de ses emplois industriels entre 1990 et 1994, essentiellement dans la partie Est), et plus encore.

Une stratégie de marketing urbain concertée atteint désormais des dimensions jusquelà inconnues, avec un professionnalisme jusqu'alors inédit symbolisé par la création de la Holding Partner für Berlin en 1994. "Le nouveau Berlin " (das neue Berlin) devient la devise de la nouvelle ère - simple et pourtant presque messianique dans sa promesse. La reconstruction s'est accompagnée d'un nouveau récit : la ville qui renaît de ses cendres, la nouvelle capitale de l'Europe, le pont entre l'Orient et l'Occident, la " ville verte ». Les grands projets nécessitent de grands gestes.

La construction de la Potsdamer Platz fut probablement le projet le plus emblématique des années 1990, même si son importance en termes architecturaux aujourd'hui est discutable. Une grande superficie d'environ 7 hectares adjacente au ur de Berlin a été vendue à de grands promoteurs associés à des conglomérats industriels : Daimler Benz, Sony, ABB. Un nouveau plan directeur, établi par l'architecte Renzo Piano, a défini les contours principaux du projet : haute densité, mixité avec un accent mis sur les espaces commerciaux et les bureaux, les grands complexes de divertissement (cinémas, théâtre, casino) et les architectes vedettes. Le déménagement du très symbolique Festival du film de Berlin (Berlinale) sur la Potsdamer Platz quelques années plus tard, à proximité de la Philharmonie de Berlin, lui a néanmoins donné un supplément d'âme.

Mais loin des grands gestes urbains et des paillettes, l'économie de la ville vacillait. La base industrielle de la ville s'est effondrée à la fois à l'Est, avec la fermeture de la plupart des usines, et à l'Ouest avec la fin des subventions de l'État qui maintenaient BerlinOuest en vie depuis des décennies. La perte d'activités productives et l'énorme 
investissement dans les infrastructures ont poussé les finances de la ville à un point de rupture. Dès le milieu des années 1990, la dette publique de la Ville-Land atteignait 60 milliards d'euros. Lorsqu'en 1998-1999 la bulle mondiale d'Internet éclata, Berlin fut durement touché.

Ce qui marque également les années 1990, ce sont les grands projets de reconstruction et de réhabilitation de quartiers entiers, tant dans le centre-ville que dans sa périphérie. La durabilité passe au deuxième plan, car la " croissance " devient le principal mot d'ordre de la période. Seul le Schéma Directeur (FNP, cf. plus haut) définit les limites de la construction et la protection des espaces verts. Cependant, la « ville verte " fait partie du récit du nouveau Berlin et elle est considérée comme un avantage compétitif pour y attirer des investisseurs et des touristes. Aussi, forte de l'expérimentation de " réhabilitation douce » d'immeubles des années 1980 (cf. les réussites de " I'IBA-Neu » et de «I'IBA-Alt » à Kreuzberg et Neukölln notamment ${ }^{1}$ ), les nouvelles constructions prennent en compte l'optimisation énergétique. De plus, pour les grands terrains vagues du centre-ville repris par de grands projets, de nouveaux espaces verts de substitution sont créés qui seront ensuite transformés en parcs (par exemple, en 1997 le parc du Gleisdreieck, voir plus bas).

Cependant, avec le creusement de la dette publique, c'en est fini des grands projets. Mais pas de la "ville verte".

\section{1 - 2011 « Small is beautiful » : de la « ville sociale » à la « ville créative »}

La frénésie de dépenses des années 1990 s'est soudainement arrêtée en 2001. Un scandale bancaire à grande échelle éclata, impliquant une grande partie de la classe politique berlinoise, principalement issue du parti conservateur. La ville était au bord de la faillite, alors que le chômage montait en flèche, atteignant près de $20 \%$ en l'an 2001. Le gouvernement de l'inamovible Eberhard Diepgen (CDU) fut contraint de démissionner et un nouveau maire - le charismatique Klaus Wowereit (SPD) - prit le relais, menant une coalition transitoire entre le parti social-démocrate (SPD) et les verts (Bündnis 90/Die Grünen).

\footnotetext{
1 Dans les années 1980, Berlin-Ouest est confronté au double problème du déclin démographique et de l'appauvrissement des quartiers populaires de Kreuzberg, Neukölln, Tempelhof et Wedding. La réponse des édiles à ces deux difficultés consiste à se lancer dans un vaste programme de rénovation (IBA-Neu) et de réhabilitation (IBA-AIt) dans le cadre d'une nouvelle Exposition internationale d'architecture ou Internationale Bau-Ausstellung (IBA) dont les résultats sont présentés à l'occasion du 750ème anniversaire de la ville en 1987. À l'occasion de l'IBA sont mis au point des types d'immeubles HLM nouveaux et sont expérimentés de nouveaux matériaux, de nouvelles techniques d'isolation phonique et énergétique, etc. D'une certaine manière, on peut considérer I'IBA des années 1980 à Berlin-Ouest comme l'ancêtre de la ville durable.
} 
Sous la pression cumulée du gouvernement fédéral, de la dette et des tensions internes à Berlin, le nouveau gouvernement de la Ville-Land lança un programme sans précédent de cessions et de privatisations : logement, gaz, électricité, etc. Cependant, l'aspect le plus significatif de cette frénésie de privatisation, qui aura des conséquences à long terme à Berlin, a été la vente massive de logements sociaux à des sociétés immobilières privées.

Les élections de 2001 ont confirmé Klaus Wowereit comme maire de Berlin, et les sociaux-démocrates formèrent un gouvernement de coalition avec l'ex-PDS devenu Die Linke (extrême gauche), une coalition rouge-rouge solide qui a gouverné jusqu'en 2011, après sa réélection en 2006. Principale victime de cette coalition inédite à l'échelle de l'Allemagne : les Verts, qui se retrouvent dans l'opposition. De fait, la préoccupation majeure de la coalition rouge-rouge menée par Klaus Wowereit est d'ordre social-et économique, et peu environnementale. Mais c'est l'urgence de la situation qui l'imposait. II y a une certaine ironie historique dans le fait que c'était cette coalition de gauche qui devait achever le programme de privatisation que le gouvernement précédent avait lancé. La quasi-faillite de Berlin, la stagnation de la population, l'absence d'investissements étrangers, ainsi que le changement à la tête du ministère local de l'urbanisme (la nouvelle ministre était une spécialiste en sciences sociales),-ont conduit à un changement d'orientation majeur : les projets à grande échelle ont été abandonnés et la planification a commencé à se concentrer sur le local. Ceci est visible dans deux stratégies bien distinctes : le programme de la « ville sociale » et le récit de la "ville créative ».

Le programme de "ville sociale " (Soziale Stadt) a été mis sur pied par le gouvernement fédéral à la fin des années 1990 pour contrer la ségrégation sociale croissante qui devenait visible dans les villes (Bocquet et Laborier, 2016). Grâce à son outil principal, la "gestion de quartier" (Quartiersmanagement), il s'agissait d'un programme spatialisé qui cherchait à résoudre les problèmes sociaux au niveau du quartier. En introduisant une structure de gouvernance à petite échelle, le "gestionnaire de quartier" (Quartiersmanager), il a créé un niveau intermédiaire entre la municipalité, les citoyens et les autres acteurs locaux. Le système fonctionne de la manière suivante : dans les quartiers jugés socialement problématiques (sur la base d'une série d'indicateurs), un gestionnaire de quartier est installé avec une petite équipe, dont le rôle est de travailler avec les acteurs locaux pour résoudre les problèmes locaux et canaliser les financements de l'État. L'une des principales innovations du programme est la création d'un " conseil de quartier " (Quartiersrat) composé d'élus et d'un " fonds de quartier » (Quartiersfonds). Entendus ici au sens de durabilité sociale, les objectifs de la ville durable continuent d'être mis en avant. De fait, même si le bilan de cette politique à petite échelle apparaît très contrasté tant les résultats divergent d'un Kiez (quartier) à l'autre, l'installation d'un "gestionnaire de quartier " au cœur d'îlots à problèmes a permis de "recoudre du lien social », selon une expression à la mode à l'époque. Elle a offert à des populations en difficulté un interlocuteur de terrain (pour ne pas dire 
" tout-terrain ») qui cherchait à privilégier le lien social et à faciliter l'accès des habitants aux services publics. Mais elle n'a pas permis de résoudre les problèmes de fond : forte concentration de population immigrée ségréguée, chômage, pauvreté, délinquance et petite criminalité.

Le deuxième grand changement a été l'abandon de l'investissement étranger à grande échelle qui était au centre de la politique économique des années 1990 et un virage vers un nouveau type de projets à petite échelle : les entreprises créatives. C'est une période où l'intérêt mondial pour l'économie créative (classe créative, industries créatives, villes créatives) grandit alors que l'économie mondiale traverse une crise généralisée (cf. Landry, 2000 ; Florida, 2002, 2005). À Berlin, le ministère de l'économie commande une série de rapports sur les industries culturelles et créatives (Senatsverwaltung für Wirtschaft, Technologie und Frauen, 2005, 2008; Senatsverwaltung für Wirtschaft, Technologie und Forschung, 2013) pour cartographier ce secteur dans la ville et élaborer une politique industrielle appropriée. Ce changement s'accompagne d'une mutation démographique : Berlin rajeunit et attire des jeunes talents du monde entier. Un espace de vie et de travail abordable, une vie culturelle vibrante, un style de vie ouvert et hédoniste deviennent des éléments clés de la vie urbaine, repris sous forme de messages marketing officiels de Berlin (Kalandides, 2012). C'est le maire de Berlin luimême, Klaus Wowereit, qui donne à la ville en 2003 son nouveau slogan : " arm aber sexy " (" pauvre mais sexy »). Ce slogan fait mouche chez les créatifs de tous horizons à la recherche de locaux abordables et d'ambiance urbaine "cool» (Colomb, 2012). Symbole de cette nouvelle image, Berlin est labellisée en 2006 « ville mondiale du design » par I'UNESCO.

La protestation citoyenne, qui était bien présente mais peu visible pendant près de deux décennies, voit également une résurrection dans cette période : le 13 juillet 2008, un référendum est organisé contre les projets du grand plan d'investissement du gouvernement de l'État le long de la rivière Spree, qui est passé à la postérité sous le nom de "Mediaspree ». Le nombre requis de signatures de soutien fut atteint cinq mois plus tôt que prévu et fut soumis aux responsables de l'arrondissement de Friedrichshain-Kreuzberg. Une majorité très nette (87\%) soutint les propositions alternatives de l'initiative de protestation (" Mediaspree versenken " ou " coulez Mediaspree!»). Selon I'organisation Mehr Demokratie ("Plus de démocratie »), il s'agissait du mouvement citoyen berlinois le plus réussi à ce jour. Le référendum fut un succès de deux manières : tout d'abord, il a réussi à arrêter pour un temps le développement prévu en protégeant l'espace ouvert le long du fleuve (bien que la récession économique de l'époque ait peut-être aussi été un facteur décisif à cet égard) ; et deuxièmement, il a consolidé un mouvement social qui devait jouer un rôle important à l'avenir (voir ci-dessous avec l'exemple de l'aéroport de Tempelhof).

Un autre projet vert important est inauguré la même année 2008, le parc du Gleisdreieck, un espace vert d'environ 26 hectares, situé au sud de la Potsdamer Platz, 
de l'autre côté du Landwehrkanal. Ce couloir vert de direction nord-sud crée un système continu de sentiers depuis le Natur-Park Südgelände à Schöneberg en passant par les parcs de la Potsdamer Platz jusqu'au Tiergarten. Depuis les années 1970, il y avait eu des intentions de planification allant du développement à un projet d'autoroute. Ce sont des initiatives citoyennes qui considéraient cet endroit comme idéal en tant qu'espace récréatif qui se sont opposées à ces plans de développement - avec succès. En 1997, dans un contexte récessif, le Land de Berlin décida définitivement de consacrer cette ancienne friche aux espaces verts et à la détente.

Le parc du Gleisdreieck, conçu par l'Atelier Loidl en tenant compte des souhaits des habitants, est un véritable parc public pour différents groupes d'usagers. Les souhaits et les idées des Berlinois ont été intégrés dans la planification et la réalisation du parc dès le départ. Avant même l'annonce du concours d'aménagement paysager, il y eut un dialogue intensif avec les futurs utilisateurs via des enquêtes auprès des ménages, des échanges en ligne et des événements sur place. L'objectif principal des mesures d'entretien et de développement était d'harmoniser les intérêts de la conservation de la nature et de l'utilisation récréative dans le parc. Pendant sa période de " jachère ", une diversité écologique unique s'était développée au Gleisdreieck, qui a été soigneusement préservée dans le nouveau parc.

Ainsi, au début du nouveau siècle à Berlin, le thème de la ville durable peut être décliné à travers ses trois aspects : environnemental, avec la création de grands parcs urbains ; économique, avec la création in situ d'industries créatives; et social, avec les programmes de la ville sociale et du Quartiersmanagement.

\section{1 - 2016 : l'ère de la Smart City}

En 2011, un nouveau gouvernement, formé par une alliance entre le Parti socialdémocrate et les Chrétiens-démocrates, arrive au pouvoir. La nouvelle coalition modifie la stratégie politique de ses prédécesseurs et se concentre sur deux thématiques qui sont aussi deux nouveaux mots-clés : le concept de la "Smart city/ville intelligente", issu du débat sur les industries créatives, ainsi que le concept de "ville durable ", déjà éprouvé ailleurs, deviennent les nouveaux mantras urbains. En 2015, après plusieurs années d'élaboration, le nouveau cadre de la "Smart City/ville intelligente » est publié, censé constituer la pierre angulaire du développement de Berlin en une ville gérée par un nouveau système basé sur l'informatique. Le gouvernement de Berlin donne sa propre définition de l'idée de la durabilité : les villes sont adaptées pour l'avenir si elles atteignent un niveau de qualité de vie sensiblement plus élevé ou stable avec une utilisation des ressources identique ou inférieure. Cela ne pourrait être réalisé, affirment les documents officiels, que grâce à une gestion urbaine utilisant des technologies de l'information et de la communication innovantes qui relieraient diverses sources d'information et permettraient ainsi la création et l'utilisation de synergies augmentant 
significativement l'efficacité et la conservation des ressources, grâce à des approches intégrées. Cela englobe à la fois les processus de production, les services et les technologies ainsi que les infrastructures qui sont intégrées, mises en réseau, mutuellement soutenues et donc uniquement réalisables en premier lieu grâce aux nouvelles technologies de l'information et de la communication "intelligentes " (Senatsverwaltung für Stadtentwicklung und Umwelt, 2015). L'enthousiasme public autour de la Smart City s'est clairement atténué depuis, à mesure que les critiques à la fois académiques et politiques du concept augmentaient (Picon, 2013, Bauriedl et Strüver (dir.), 2018, Ghorra-Gobin, 2018). D'un côté, I'influence qu'auraient d'énormes conglomérats internationaux sur la ville et sur les données privées a été jugée très problématique, tandis que d'un autre côté, la foi en un optimisme technologique exagéré s'est affaiblie.

Par ailleurs, c'est au cours de ces années que la concurrence pour l'espace est devenue le plus visible, car la population de Berlin augmentait de façon constante. Cette concurrence ne s'explique pas seulement par l'attractivité retrouvée de la capitale de l'Allemagne. Elle est aussi une conséquence de la crise financière mondiale de 20072008, lorsque l'investissement dans l'immobilier est devenu l'un des rares paradis pour le capital accumulé dans l'industrie ou les affaires. À la fin des années 2000, Berlin est en effet la dernière capitale européenne abordable. Les loyers y sont très modiques et le prix d'achat se situe en moyenne autour de $1200,-€$ au mètre carré $(3000,-€$ aujourd'hui). La ville représente alors un véritable eldorado pour les investisseurs internationaux, fonds de pension, promoteurs et grands groupes immobiliers qui rachètent des dizaines de milliers de mètres carrés d'appartements, d'immeubles, de bureaux ou de friches. De son côté, la ville, toujours très endettée, a un gros besoin d'argent. Par le biais des sociétés immobilières publiques qu'elle gère (Wohnungsbaugesellschaften), elle cède ainsi au privé quantité de terrains, bâtiments ou logements sociaux. On assiste alors, de manière silencieuse, à un gigantesque transfert de biens fonciers et immobiliers de la puissance publique vers le capital privé. À ce niveau-là, la réalité vient brutalement contredire le mythe de la ville sociale durable. Les habitants concernés par ce transfert et par l'augmentation des loyers qui en découle ne tarderont pas à s'en rendre compte (Holm, 2014 ; Häussermann et alii, 2002).

La pression exercée sur la ville de Berlin pour utiliser de plus en plus de nouveaux terrains pour la construction, pourtant limitée par le plan d'aménagement du territoire de 1994, se manifeste dans certains cas emblématiques, comme celui de l'ancien aéroport de Tempelhof, situé au Sud du centre-ville mais à l'intérieur de la ceinture ferroviaire du S-Bahn, autrement dit à l'intérieur de I'Innenstadt (partie centrale de l'agglomération). En raison de sa non-conformité aux normes internationales et de la construction du principal aéroport de Berlin-Brandenburg International (BBI), l'aéroport de Tempelhof est fermé en 2008. À sa place, le Tempelhofer Feld (selon le nom du terrain en question) est inauguré en 2010. II devient vite une zone de promenade et de 
détente de 355 hectares qui couvre la totalité de l'espace de l'ancien aéroport (bâtiments, pistes d'atterrissage, tarmac, etc.). Cet espace de loisirs devient ainsi le plus grand espace ouvert de centre-ville, à la fois le plus grand parc urbain de Berlin et l'un des plus grands d'Europe.

Déjà en juin 2009, plusieurs milliers d'activistes qui avaient formé une alliance appelée "Squat Tempelhof » avaient tenté d'occuper le site pour protester contre les plans de construction du terrain. Les manifestants critiquaient le fait que la zone n'avait pas été ouverte au public et craignaient, parallèlement à la privatisation et à la commercialisation croissantes du foncier, que le processus de gentrification ne s'accélère.

En septembre 2011, une initiative citoyenne appelée "100\% Tempelhofer Feld " fut fondée dans le but de bloquer les plans du Sénat pour d'autres utilisations ultérieuress, au moyen d'un référendum, et d'empêcher toute construction sur le site. D'après la présentation de l'initiative, l'espace ouvert devait être entièrement préservé pour le public et ne pas être doté des nouveaux bâtiments prévus dans les plans du Sénat, comme la nouvelle bibliothèque d'État, les logements sociaux et les propriétés résidentielles et commerciales, ni être associé à l'Exposition internationale des jardins de 2017. Des signatures contre ce projet ont été recueillies en masse, ce qui a obligé le Sénat à autoriser la tenue d'un référendum. Ce dernier a eu lieu en mai 2014 et a été couronné de succès, avec une nette majorité de voix contre toute construction sur le site et pour sa préservation tel quel, en tant qu'espace ouvert à la population. Le maire de Berlin, Klaus Wowereit, n'eut pas d'autre choix que d'accepter le résultat des urnes.

Ce référendum citoyen fut rapidement qualifié d'historique non seulement par l'ampleur du terrain qu'il concernait mais aussi du fait de la prise de conscience par une majorité de Berlinois de l'importance vitale, dans une ville en plein boom, des espaces verts et ouverts. Auparavant, dans les années 1990, d'autres initiatives citoyennes s'étaient battues avec succès pour la préservation du poumon vert du centre-ville, le Tiergarten, menacé sur ses franges par le grand projet de tunnel Nord-Sud passant sous le parc. Par ce type d'initiatives comme celles de Tempelhof et du Tiergarten, et par bien d'autres encore, quoique moins médiatisées, la population manifeste son attachement constant à des espaces verts et des espaces ouverts, loin des plans d'urbanisme officiels et des incantations à la "ville verte", la "ville durable" ou encore la "ville intelligente ». Elle manifeste également son intention d'être partie prenante dans les débats sur l'avenir du cadre urbain. 


\section{Après 2016 - La question du logement et la ville verte}

La nouvelle coalition entre le SPD, les Verts et Die Linke arrivée au pouvoir fin 2016 convint d'un programme commun dans l'« accord de coalition 2016-2021» (Koalitionsvereinbarung 2016). Déjà le sous-titre du rapport - « pour un Berlin solidaire, durable, ouvert sur le monde "- est programmatique dans le sens où il définit les trois valeurs principales qui régissent l'ensemble du document. La durabilité est présente tout au long du texte et concerne tous les aspects du développement urbain : logement, énergie, investissement, économie, environnement, transports, mobilité, tourisme, etc. De plus elle est spécifiquement mentionnée dans quatre sections différentes du document : Développement urbain à Berlin - intelligent, durable et participatif (" une stratégie intégrée qui intègre les aspects sociaux, écologiques et économiques dans un équilibre durable »); une politique économique innovante, équitable et durable pour Berlin ( « La coalition s'oriente également vers les objectifs convenus dans l'accord de Paris sur le climat. Une politique économique socialement et écologiquement responsable renforce la compétitivité de Berlin »); Berlin - pionnier de la protection du climat et de la transition énergétique ( $"$ Le Land de Berlin mettra en œuvre de manière ambitieuse son objectif de devenir climatiquement neutre d'ici 2050 » ; Nature active et protection de l'environnement - un nouveau départ écologique pour Berlin («Pour la coalition, la durabilité est une tâche transversale pour tous les domaines politiques »). Au moins sur le plan programmatique, Berlin semble voué au développement urbain durable.

Cependant, le plus grand défi à Berlin est aujourd'hui la " concurrence foncière ", c'està-dire la lutte pour l'usage des terrains à long terme. Berlin est maintenant une ville en pleine croissance avec une population de 3,7 millions d'habitants et (avant la pandémie COVID-19) avec 14 millions de touristes par an, ce qui en fait la troisième destination touristique d'Europe après Paris et Londres, avant Rome, Barcelone, Madrid et Venise (Kalandides, 2020). Le logement devient de plus en plus rare, voire inabordable pour de larges segments de la population. Et bien que le nouveau gouvernement de coalition rouge-rouge-vert ait fait de l'accès à des logements abordables l'un de ses objectifs centraux, ce processus est lent et porte la cohésion sociale de la ville à ses limites. L'équilibre déjà fragile entre développement durable et croissance devient de plus en plus tendu. Pour éviter qu'il ne rompe, le Sénat de Berlin a adopté en 2019 la mesurephare portée par Katrin Lompscher, sénatrice au logement et à la construction (Die Linke), à savoir le fameux Mietdeckel qu'on peut traduire littéralement par " couvercle mis sur les loyers ». Dans les faits, il s'agit bien d'une mesure révolutionnaire puisqu'elle consiste à interdire l'augmentation des loyers pendant cinq ans, sauf dans des proportions très modestes fixées par la loi. Même si la validité juridique de cette loi, qui fut tout de suite contestée par les syndicats de propriétaires et par les promoteurs, doit encore être examinée par la Cour constitutionnelle de Karlsruhe, il est peu probable que le Sénat la retire purement et simplement, y compris pour des raisons électorales (les prochaines élections municipales se tiendront en septembre 2021). En effet, dans un 
contexte économique très tendu et incertain, le Mietdeckel compte de plus en plus de partisans parmi les habitants. Dans une certaine mesure, on peut même aller jusqu'à affirmer qu'il incarne mieux que tout autre règlement le "Berlin solidaire, social et durable » que la coalition au pouvoir appelait de ses vœux.

Cette concurrence croissante sur le terrain a consolidé les mouvements sociaux urbains qui tentent de sanctuariser l'accès à certains bâtiments ouverts et certains espaces verts de la ville. Ainsi - à côté de Tempelhof ou des rives de la Spree (Spreeufer) - d'autres sites comme la Haus der Statistik, le Dragonerareal ou le Mauerpark (voir plus loin) sont devenus des espaces de litiges ou de conflits entre une intensification des investissements et le besoin d'avoir des espaces accessibles pour couvrir les besoins économiques et sociaux ainsi que les activités de détente et de loisirs. Les " jardins communs " peuvent être conçus comme de tels espaces qui à la fois résistent à la pression de la construction et répondent à un nouveau sens de la communauté. La notion de "Vert urbain productif» (Produktives Stadtgrün) adoptée par le Sénat combine les jardins familiaux, l'agriculture urbaine et l'autosuffisance avec la culture du do-it-yourself et un style de vie écologiquement motivé2 ${ }^{2}$

Avec environ 200 jardins communs en septembre 2019, Berlin est devenu au cours des dix dernières années un centre de jardinage communautaire urbain important à l'échelle de l'Europe. Bien sûr, les jardins familiaux et ouvriers du début du XXème siècle constituaient déjà une première forme de jardinage urbain, mais il s'agissait de parcelles individuelles et non pas de jardins partagés. Le jardinage communautaire a donné une toute nouvelle impulsion à la signification sociale et à la perception publique des espaces ouverts utilisés pour le jardinage dans la ville.

Le jardin communautaire interculturel Allmende-Kontor sur l'ancien aéroport de Tempelhof, exploité par plus de 500 jardiniers depuis 2011, est un projet emblématique de ce mouvement. Sur $5000 \mathrm{~m}^{2}$, il permet aux personnes sans jardin, en particulier celles issues des quartiers socialement défavorisés de Neukölln ou des arrondissements voisins, de jardiner ensemble ou simplement d'être au grand air. Les Prinzessinnengärten sont probablement le projet de jardinage urbain le plus célèbre de Berlin. II s'agit d'un jardin mobile sur la Moritzplatz, dans le quartier populaire de Kreuzberg, qui a été créé en 2009 à l'initiative de résidents et est géré par l'association à but non lucratif Nomadisch Grün. Une ancienne friche a été convertie en potager pour l'agriculture urbaine. L'espace est loué annuellement par la ville et le but est de convertir un terrain vague en un lieu de " verdure productive " ainsi que d'apprentissage et de création communs.

Enfin, après plus de deux ans de processus de participation étendue entre les administrations d'arrondissement et le Sénat, les associations et la société civile, la

2 https://www.berlin.de/gemeinschaftsgaertnern/ 
"Charte de la ville verte de Berlin" (Senatsverwaltung für Umwelt, Verkehr und Klimaschutz, 2020) a été approuvée par le Sénat au début de l'année 2020. Ce document en forme de manifeste vise à préserver et augmenter la valeur écologique de la ville en développant ses espaces verts et naturels. Reste à observer si, à une époque rendue incertaine par le coronavirus, ses multiples conséquences et les changements de priorités qu'il implique, la Charte deviendra réalité ou restera un vœu pieux.

Le Mauerpark résume sans doute mieux que beaucoup d'autres jardins les péripéties des friches du centre-ville de Berlin. À l'origine un grand site ferroviaire d'avant-guerre, occupé ensuite par le Mur de Berlin, il fait l'objet de controverses depuis des décennies. Le Mauerpark est coincé entre trois quartiers très distincts : sur l'ancien côté occidental du Mur, le quartier de Wedding, avec une forte présence de communautés ouvrières et de migrants, et sur l'ancien côté Est, d'une part Prenzlauer Berg, peut-être le quartier le plus embourgeoisé de Berlin et d'autre part, le quartier branché et touristique de Mitte. Le Mauerpark (littéralement le "parc du Mur ") est ainsi intensément utilisé par tous les différents groupes représentés dans les zones adjacentes - touristes compris - créant parfois des conflits avec les riverains. Cependant, le plus grand conflit de ces dernières années a été celui entre les logements privés et le jardin. Une partie de la zone a été vendue à des promoteurs privés qui ont construit de nouveaux complexes de logements haut de gamme (et certains abordables). Les tentatives de mobilisation citoyenne et de participation à la prise de décision ont avorté lorsque le Sénat et les promoteurs se sont rapidement entendus afin que la construction de ces nouveaux logements en bordure de parc commence. Aujourd'hui, ils ont vu le jour mais la plus grande partie du Mauerpark reste un parc soigneusement aménagé qui accueille toujours toutes les différentes communautés locales et de plus en plus de touristes.

\section{CONCLUSION :}

Même si Berlin s'est doté dès les années 1990 d'un arsenal de règles, de principes et d'objectifs pour devenir une "ville verte et durable ", force est de constater qu'elle n'est pas parvenue, dans un premier temps, à donner corps et réalité à ces principes. A l'inverse des villes allemandes pionnières en matière d'écologie et de durabilité, telles Fribourg-en-Brisgau ou Tübingen, et des autres grandes métropoles du pays telles Munich, Hambourg ou Francfort, Berlin ne fait pas de la ville verte et des transports propres sa priorité durant les années 1990. À sa décharge, la ville a à gérer d'autres problèmes, plus urgents : une économie en grande difficulté, un appareil industriel en faillite, un chômage endémique, une réunification problématique. En outre, les espaces verts et ouverts, dans le Berlin des années 1990 et 2000, sont loin de constituer une denrée rare. Au contraire, les friches urbaines issues de la déprise industrielle, de l'ancien Mur et de la destruction d'immeubles ou d'usines vétustes, viennent s'ajouter à la profusion des espaces verts et ouverts dans la ville. 
Il faut attendre une dizaine d'années avant d'observer les premiers résultats concrets du plan "ville verte et durable ". C'est au tournant des années 1990 et 2000 que de nouveaux parcs de centre-ville sont livrés : le parc du Gleisdreieck au Sud de la Potsdamer Platz et le Südgelände en continuité avec le premier, en fournissent de bonnes illustrations. Ils furent d'ailleurs aussitôt adoptés par la population. C'est également à cette époque que la Ville-Land se dote de nouvelles réglementations en matière d'espaces publics, de construction de logements, d'isolation thermique, etc. Le terme de "ville durable " s'ancre alors dans le vocabulaire urbain. Las, l'endettement de la ville est tel qu'il condamne celle-ci à brader des milliers de logements municipaux à des sociétés immobilières privées, au premier rang desquels la Deutsche Wohnen, tandis qu'investisseurs internationaux, promoteurs et fonds de pension se positionnent autour du juteux marché que représentent les logements de la capitale allemande redevenue attractive.

Les prix de l'immobilier explosent dans les années 2010, la ville sociale durable a vécu. En revanche, il existe une vraie durabilité, plutôt une vraie continuité dans les tensions liées au manque de logements abordables. Des conflits parfois violents éclatent dans les arrondissements de Kreuzberg et de Neukölln, les derniers squats sont expulsés. Partout, les Berlinois se battent pour conserver des logements pas chers, des infrastructures de qualité et des espaces verts de proximité. C'est certainement sur ce dernier plan qu'ils obtiennent le plus de victoires, la plus significative étant incontestablement le succès du référendum de 2014 sur le maintien en l'état du terrain de l'ex-aéroport de Tempelhof.

Aujourd'hui, leurs revendications semblent avoir été en partie entendues par les politiques au pouvoir. La coalition "rouge-rouge-verte» au pouvoir depuis 2016 a pris en effet un certain nombre de mesures qui vont toutes dans le sens d'une ville durable et verte : protection des loyers pendant cinq ans (Mietdeckel), construction de nouvelles pistes cyclables le long des grands axes de circulation (une vieille revendication des habitants), piétonnisation de secteurs stratégiques en centre-ville (Friedrichstrasse à l'Est, Breitscheidplatz / une partie du Kurfürstendamm à l'Ouest), renouvellement du parc de bus (passage à l'hybride ou à l'électrique), de trams, de métros et de S-Bahn, fermeture définitive de l'aéroport de Tegel et transformation prévue en éco-quartier (ce point reste à confirmer), sanctuarisation de presque tous les espaces verts et parcs de la ville, reconnaissance progressive des micro-jardins et des jardins partagés, normes contraignantes adoptées en matière de réduction des polluants et du $\mathrm{CO} 2$, objectif de ville neutre en 2050 confirmé. Il ne s'agit plus d'un catalogue de bonnes intentions tel qu'on pouvait le relever au début de la période mais bien de mesures concrètes déjà appliquées ou en passe de l'être.

Ainsi, progressivement, le slogan de la ville durable et verte s'est imposé à la fois dans les mentalités et dans l'agenda politique. Cependant, il convient de souligner que Berlin, malgré ses efforts et ses spécificités, ne fait que s'aligner sur des standards 
métropolitains internationaux (Grésillon, 2017) et qu'elle tente de rattraper son retard sur d'autres villes européennes, à l'instar de Copenhague, Stockholm ou Rotterdam, qui sont nettement plus en avance sur le plan de la durabilité.

\section{Eléments de bibliographie :}

Abgeordnetenhaus von Berlin (dir.), Gesetz zur Umsetzung der Energiewende und zur Förderung des Klimaschutzes in Berlin vom 22. März 2016, Berlin, 2016.

Bauriedl, Sibylle, Strüver, Anke, Smart City-Kritische Perspektiven auf die Digitalisierung in Städten, Berlin, Transcript-Verlag, 2018.

Bocquet, Denis, Laborier, Pascale, Sociologie de Berlin, Paris, La Découverte, coll. "Repères", 2016.

Colomb, Claire, Staging the new Berlin, Londres, Routledge, 2012.

Florida, Richard, The Rise of the Creative Class, New York, Basic Books, , 2002.

Florida, Richard, The Flight of the Creative Class, New York, Harper Business, 2005.

Ghorra-Gobin, Cynthia, "Smart City : 'fiction' et innovation stratégique", in Quaderni, 2018-2, n 96, p. 5-15, 2018.

Grésillon, Boris, "Villes créatives" vs. "villes de création" ?, in Liefooghe C. (dir.), L'économie créative et ses territoires, Rennes, Presses universitaires de Rennes, 2014.

Grésillon, Boris, "Berlin sur la voie de la normalisation ? Essai de psychogéographie", in Allemagne d'aujourd'hui, dossier spécial sur Berlin coordonné par B. Grésillon et S. Vannier, n²21, été 2017, p. 39-56, 2017.

Häussermann, Hartmut et alii, Stadterneuerung in der Berliner Republik. Modernisierung in Berlin-Prenzlauer Berg, Reihe: Stadt, Raum und Gesellschaft, vol. 16, Opladen, Leske und Budrich, 2002.

Holm, Andrej, Mietenwahnsinn. Warum Wohnen immer teurer wird und wer davon profitiert, Munich, Droemer Knaur, 2014.

Kalandides, Ares, "Berlin : les industries créatives et l'aménagement urbain", in Terrin J.J. (dir.), La ville des créateurs, Marseille, Parenthèses, p. 30-49, 2012.

Kalandides, Ares, "Tourism Management in Berlin: From Destination Marketing to Place Management", Oskam, J.A. (Dir.) The Overtourism Debate, Emerald Publishing Limited, p. 251-265, 2020.

Koalitionsvereinbarung zwischen Sozialdemokratische Partei Deutschlands (SPD) Landesverband Berlin, DIE LINKE Landesverband Berlin und BÜNDNIS 90/ Die Grünen 
Landesverband Berlin für die Legislaturperiode 2016-2021. (2016) [sans informations bibliographiques] https://www.berlin.de/rbmskzl/regierenderbuergermeister/senat/koalitionsvereinbarung/

Landry, Charles, Creative City. A Toolkit for urban Innovators, Earthscan, 2000.

Picon, Antoine, Smart Cities: Théorie et critique d'un idéal auto-réalisateur, Édition B2, 2013.

Senatsverwaltung für Stadtentwicklung und Umwelt (dir.), Smart City-Strategie Berlin, Berlin, 2015.

Senatsverwaltung für Stadtentwicklung und Umwelt (dir.), Berliner Nachhaltigkeitsprofil Berliner Potenziale und Begabungen für die nachhaltige Entwicklung nutzen, Berlin, 2016.

Senatsverwaltung für Umwelt, Verkehr und Klimaschutz (dir.), BEK 2030. Berliner Energie- und Klimaschutzprogramm 2030, Berlin, 2019.

Senatsverwaltung für Umwelt, Verkehr und Klimaschutz (dir.), Charta für das Berliner Stadtgrün, Berlin, 2020.

Senatsverwaltung für Wirtschaft, Technologie und Frauen (dir.), Kulturwirtschaft in Berlin. Entwicklung und Potenziale, Berlin, 2005.

Senatsverwaltung für Wirtschaft, Technologie und Frauen (dir.), Kulturwirtschaft in Berlin. Entwicklung und Potenziale, Berlin, 2008.

Senatsverwaltung für Wirtschaft, Technologie und Forschung (dir.), Dritter Kreativwirtschaftsbericht. Entwicklung und Potenziale, Berlin, 2013. 